\title{
Antimicrobial Susceptibility of Escherichia coli Strains Collected from the Southwestern Coast of Istanbul
}

\author{
Nuket Sivri* and Vildan Akbulut \\ Department of Environmental Engineering, Faculty of Engineering, \\ Istanbul University 34320, Avcýlar, Istanbul, Turkey. \\ http://dx.doi.org/10.13005/bbra/2098
}

(Received: 01 April 2016; accepted: 19 May 2016)

\begin{abstract}
Antimicrobial resistance is one of the biggest problems facing global public health. Among the microorganisms with antimicrobial resistance, Escherichia coli species strains out due to its dual role as fecal contamination indicator and pathogen. In this study was found that the prevalence's of antimicrobial resistance (AR) and multiple antimicrobial resistance (MAR) of Escherichia coli in the southwestern coast of Istanbul, under strong anthropogenic pressures, for a period of three years starting in January 2009 through December 2011. The fecal indicator bacterium, Escherichia coli, was tested for their susceptibility to different group of 10 antimicrobials, using the disk diffusion method. In this study, 194 strains of E.coli were isolated, in which ampicillin $(\mathbf{7 4 . 4 \% )}$ and amoxicillin $(47.4 \%)$ had the highest resistance rates. Tetracycline resistance was found to be $43.3 \%$. While $84.4 \%$ of the isolates were found to be resistant to at least one or more antibiotic, $63.4 \%$ were resistance to 2 or more antibiotics and $24.7 \%$ were resistant to 5 or more antibiotics. In addition, no resistance was detected in the antibiotic imipenem. With the number of the resistant strains out numbering the number of sensitive strains, serious concerns regarding antibiotic resistance in sea based bacteria are raised. As a result, the association between wrong and insufficient refinement and pollution indicator bacteria spreading throughout the environment is clear. These findings, which were obtained throughout Istanbul's shores which are frequently used for recreation, fishing, and transport, show that public health is under serious risks.
\end{abstract}

Key words: Escherichia coli, antibiotic resistance, multiple antibiotic resistances, Istanbul, Turkey

Antibiotics are probably the most successful family of drugs that have been developed to improve human health. In addition to this fundamental use, antibiotics (antimicrobials at large) have also been used for preventing and treating humans, animals and plants infections as well as for promoting growth in animal farming ${ }^{1}$. In all of these applications, antibiotics, as well as their residues, are continuously discharged with wastewater municipal sewage systems ${ }^{2}$ which in return has increased the introduction of antimicrobial agents into the environment and this has resulted in the development of selective

\footnotetext{
* To whom all correspondence should be addressed. Tel.: + 90 (212) $4737070 / 17651$,

Fax: + 90 (212) 4737180.

E-maila : nuket@istanbul.edu.tr / sivrin@gmail.com
}

pressures on bacterial populations ${ }^{3}$.

Bacteria may develop a resistance to antibiotics under selective pressures or they may acquire antibiotic resistance determinants without direct exposure to any antibiotic ${ }^{4-6}$. Genetic coding for antibiotic resistance can spread via mobile genetic elements like plasmids, transposons and integrons ${ }^{7}$. In addition, this may also contribute to antimicrobial agent resistance in humans through acquisition via the human food chain, poor hygiene conditions, and overcrowded living conditions ${ }^{8}$. This may pose a serious threat to public health in that more and more infections may no longer be treatable with known antibiotics. In the event that antibiotic resistance spreads from nonpathogenic to pathogenic bacteria, epidemics may result ${ }^{9}$.

In aquatic environments, the presence of 
antibiotic resistant pathogen bacteria belonging to the Enterobacteriaceae is of particular concern ${ }^{10}$. Escherichia coli (E. coli), a member of the Enterobacteriaceae family, is a common inhabitant of the human and animal gut. E.coli is widely disseminated in the environment through human and animal feces and its presence in aquatic environments is generally indicative of fecal contamination ${ }^{4}$. This is the most common cause of gram-negative nosocomial and communityacquired infections ${ }^{11}$. Patterns of antibiotic resistance have been used to identify fecal pollution in water. Recent studies have shown the existence of major sources of fecal water pollution, which can be determined by conducting a Multiple Antibiotic Resistance (MAR) analysis ${ }^{12}$ or as it is now known to be Antibiotic Resistance Analysis (ARA).

The goal of this study was to investigate multiple antibiotic resistance among $E$. coli isolates collected from 7 different stations in the southwestern coast of Istanbul on a monthly basis between the years 2009, 2010, and 2011.

\section{MATERIALAND METHODS}

\section{The Study Area}

In this research, Istanbul was selected as the study area. Seven sampling stations (Figure 1) were selected along the southwestern coastline in Istanbul, which are located in highly urbanized and commercial port areas. For instance, the first station (Atakoy) was located near a harbor. The second station (Yesilkoy) was selected because it was located at the very far end of the sampling area, outside of the gulf, and because it was unaffected from the presence of blooms. Station 3 (Menekse) was selected as a region because it is used as a public beach during the summer months. The $4^{\text {th }}$ and $5^{\text {th }}$ Stations were selected in areas of the Kucukcekmece Lagoon, which is connected to the Sea of Marmara via a narrow channel, so that the lagoon ecosystem and its connection with the sea could be represented. Station 6 (Avcýlar) was used in sea transport and Station 7 (Zeytinburnu) was under the influence of domestic settlement.

\section{Sample Collection}

Water samples were obtained monthly from the seven stations along the southwestern coast of Istanbul for a period of three years starting in January 2009 through December 2011. Pre-noon water samples were collected approximately $30 \mathrm{~cm}$ below the water surface with a bacteriologic sampler (500 ml) in sterilized dark glass bottles (13). Samples were analysed under standard laboratory conditions and processed within $2 \mathrm{~h}$ of collection. The physical parameters assessed were temperature, $\mathrm{pH}$, salinity and dissolved oxygen (in situ) using a multiprobe model (Hanna Company, HI 9828).

Isolation and Identification of Bacterial Isolates

The bacteriological analyses started within 2 hours after the sample was collected. Multiple-Tube Fermentation Technique (five replicate) (9221) / Standard Total Coliform Fermentation Technique (Lauryl Sulfate Broth (Merck 1.10266)) was used to estimate the prevalence of faecal coliform bacteria and for the isolation of E. coli (9221B) ${ }^{6,13,14,15}$. Standard water and wastewater methods were applied for the analysis (9221) (13). Lactose-fermenting colonies were further characterized by replica-plating on Eosin methylene blue agar (EMB, Merck 1.01347) and enteric chromagar (HiMedia, MV1353) and incubated at $37^{\circ} \mathrm{C}$ overnight. E.coli, pink colonies, was selected on the plate and inoculated into E.coli chromagar (HiMedia, MV1353) at $37^{\circ} \mathrm{C}$ overnight. For the identification of Enterobacteriaceae species, in particular E.coli, colonies were selected and confirmed by IMVIC tests (9221F) $)^{6,13,14,15}$.

\section{Antimicrobial Susceptibility Testing}

The minimum inhibition concentration was determined by using the disk diffusion method in the Mueller-Hinton medium in accordance with the Clinical and Laboratory Standards Institute (CLSI) guidelines $(15,16)$. This method allows for the determination of total level antibiotic resistance from typical colonies by detecting the most resist phenotypes present. Ten antimicrobial agents were selected as representatives of important classes of antimicrobials: ampicillin (AM, $10 \mu \mathrm{g}$ ), amoxicillin (AMC, $30 \mu \mathrm{g}$ ), tetracycline (TE, $30 \mu \mathrm{g}$ ), chloramphenicol (C, $30 \mu \mathrm{g})$, nalidixic acid (NA, 30 $\mu \mathrm{g})$, amikacin (AK, $30 \mu \mathrm{g})$, streptomycin (S, $10 \mu \mathrm{g})$, imipenem (I, $10 \mu \mathrm{g})$, and ceftazidime (CAZ, $30 \mu \mathrm{g})$. The investigated antimicrobial agents are listed in Table 1.

The results were separately interpreted by using breakpoints indicated in CLSI guidelines for Enterobacteriaceae and non-fermenters $(15,16)$. 
Multiple antibiotic resistance (MAR) indices for individual isolates were calculated by dividing the number of antibiotics, to which the isolate was resistant, by the total number of antibiotics to which the isolate was exposed, as described previously. Isolates resistant to 3 or more chemical classes of antibiotic were considered as the multiple antibiotic resistance (MAR). The antibiotics resistances are expressed as percentages ${ }^{15}$.

\section{RESULTS}

Antibiotic resistance ratios obtained from 58, 67, and 69 strains of E.coli were tested for antibiotic sensitivity with chosen antibiotics in 2009, 2010, and 2011 are given in Figure 2 respectively. Irrespective of the year, among all E.coli isolates (194 strains), the highest resistance ratios were found in ampicillin ( $74.7 \%$ - 145 isolates) followed by amoxicillin (47.4\% - 92 isolates). Tetracycline resistance ratio was found to be $43.3 \%$ - 84 of the isolates. In addition, the following ratios for the following antibiotics were also found: nalixicid acid $36.1 \%$ - 70 isolates; trimethoprimsulphamethoxazole 33\% - 64 isolates; streptomycin
$14 \%$ - 27 isolates; ceftazidime $12.4 \%$ - 24 isolates; chloramphenicol $12.4 \%$ - 24 isolates; and amicasin $2.6 \%$ - 5 isolates. No imipemen resistance was found in any of the strains.

While $84.5 \%$ of the analyzed E.coli isolates were found to be resistant to one or more antibiotic (R1-R8), 24.7\% showed resistance to 5 or more (R5+R6+R7+R8) antibiotics. In 2009, all of the E.coli isolates showed antibiotic resistance to

Table 1. Antimicrobial agents and classes

\begin{tabular}{lll}
\hline Class & Code* & Name \\
\hline Aminoglycoside & AK & Amikacin \\
& S & Streptomycin \\
Carbapenem & I & Imipenem \\
Cephalosporin & CAZ & Ceftazidime \\
Penicillins ( $\beta$-laktam) & AM & Ampicillin \\
& AMC & Amoxicillin \\
Tetracyclines & TE & Tetracycline \\
Folate AKtagonist & SXT & Trimethoprim/ \\
& & Sulfamethoxazole \\
Quinolone & NA & Nalidixic Acid \\
Amphenicol & C & Chloramphenicol
\end{tabular}

* Code of Antimicrobial Agents

Table 2. Associated and cross resistance among E.coli isolates $(\mathrm{n}=194)$

\begin{tabular}{|c|c|c|c|c|c|c|c|c|c|c|}
\hline \multirow{2}{*}{$\begin{array}{l}\text { No of isolates } \\
\text { resistant to } \\
\text { antimicrobial agent }\end{array}$} & \multicolumn{10}{|c|}{ No (\%) of E.coli resistant strains among the isolates resistant other antimicrobial agents } \\
\hline & $A M$ & $A M C$ & $T E$ & $N A$ & $S X T$ & $S$ & CAZ & C & $A K$ & $I$ \\
\hline AM $(n=145)$ & $\begin{array}{c}145 \\
(74.7 \%)\end{array}$ & $\begin{array}{c}88 \\
(45.9 \%)\end{array}$ & $\begin{array}{c}74 \\
(38.1 \%)\end{array}$ & $\begin{array}{c}62 \\
(32 \%)\end{array}$ & $\begin{array}{c}60 \\
(31 \%)\end{array}$ & $\begin{array}{c}31 \\
(16 \%)\end{array}$ & $\begin{array}{c}21 \\
(10.8 \%)\end{array}$ & $\begin{array}{c}21 \\
(10.8 \%)\end{array}$ & $\begin{array}{c}4 \\
(2 \%)\end{array}$ & $\begin{array}{c}0 \\
(0)\end{array}$ \\
\hline AMC $(n=92)$ & $\begin{array}{c}88 \\
(45.9 \%)\end{array}$ & $\begin{array}{c}92 \\
(47.4 \%)\end{array}$ & $\begin{array}{c}60 \\
(31 \%)\end{array}$ & $\begin{array}{c}53 \\
(27.3 \%)\end{array}$ & $\begin{array}{c}51 \\
(26.3 \%)\end{array}$ & $\begin{array}{c}25 \\
(12.9 \%)\end{array}$ & $\begin{array}{c}12 \\
(6.2 \%)\end{array}$ & $\begin{array}{c}20 \\
(10.3 \%)\end{array}$ & $\begin{array}{c}2 \\
(1 \%)\end{array}$ & $\begin{array}{c}0 \\
(0)\end{array}$ \\
\hline $\mathrm{TE}(\mathrm{n}=84)$ & $\begin{array}{c}74 \\
(38.1 \%)\end{array}$ & $\begin{array}{c}60 \\
(31 \%)\end{array}$ & $\begin{array}{c}84 \\
(43.3 \%)\end{array}$ & $\begin{array}{c}45 \\
(23.2 \%)\end{array}$ & $\begin{array}{c}49 \\
(25.3 \%)\end{array}$ & $\begin{array}{c}30 \\
(15.5 \%)\end{array}$ & $\begin{array}{c}12 \\
(6.2 \%)\end{array}$ & $\begin{array}{c}22 \\
(11.3 \%)\end{array}$ & $\begin{array}{c}4 \\
(2 \%)\end{array}$ & $\begin{array}{c}0 \\
(0)\end{array}$ \\
\hline NA $(n=70)$ & $\begin{array}{c}62 \\
(32 \%)\end{array}$ & $\begin{array}{c}53 \\
(27.3 \%)\end{array}$ & $\begin{array}{c}45 \\
(23.2 \%)\end{array}$ & $\begin{array}{c}70 \\
(36.1 \%)\end{array}$ & $\begin{array}{c}43 \\
(22.2 \%)\end{array}$ & $\begin{array}{c}22 \\
(11.3 \%)\end{array}$ & $\begin{array}{c}12 \\
(6.2 \%)\end{array}$ & $\begin{array}{c}17 \\
(8.8 \%)\end{array}$ & $\begin{array}{c}5 \\
(2.6 \%)\end{array}$ & $\begin{array}{c}0 \\
(0)\end{array}$ \\
\hline SXT $(n=64)$ & $\begin{array}{c}60 \\
(31 \%)\end{array}$ & $\begin{array}{c}51 \\
(26.3 \%)\end{array}$ & $\begin{array}{c}49 \\
(25.3 \%)\end{array}$ & $\begin{array}{c}43 \\
(22.2 \%)\end{array}$ & $\begin{array}{c}64 \\
(33 \%)\end{array}$ & $\begin{array}{c}28 \\
(14.4 \%)\end{array}$ & $\begin{array}{c}10 \\
(5.2 \%)\end{array}$ & $\begin{array}{c}18 \\
(9.3 \%)\end{array}$ & $\begin{array}{c}3 \\
(1.5 \%)\end{array}$ & $\begin{array}{c}0 \\
(0)\end{array}$ \\
\hline$S(n=27)$ & $\begin{array}{c}31 \\
(16 \%)\end{array}$ & $\begin{array}{c}25 \\
(12.9 \%)\end{array}$ & $\begin{array}{c}30 \\
(15.5 \%)\end{array}$ & $\begin{array}{c}22 \\
(11.3 \%)\end{array}$ & $\begin{array}{c}28 \\
(14.4 \%)\end{array}$ & $\begin{array}{c}27 \\
(14 \%)\end{array}$ & $\begin{array}{c}8 \\
(4.1 \%)\end{array}$ & $\begin{array}{c}12 \\
(6.2 \%)\end{array}$ & $\begin{array}{c}3 \\
(1.5 \%)\end{array}$ & $\begin{array}{c}0 \\
(0)\end{array}$ \\
\hline CAZ $(n=24)$ & $\begin{array}{c}21 \\
(10.8 \%)\end{array}$ & $\begin{array}{c}12 \\
(6.2 \%)\end{array}$ & $\begin{array}{c}12 \\
(6.2 \%)\end{array}$ & $\begin{array}{c}12 \\
(6.2 \%)\end{array}$ & $\begin{array}{c}10 \\
(5.2 \%)\end{array}$ & $\begin{array}{c}8 \\
(4.1 \%)\end{array}$ & $\begin{array}{c}24 \\
(12.4 \%)\end{array}$ & $\begin{array}{c}3 \\
(1.5 \%)\end{array}$ & $\begin{array}{c}2 \\
(1 \%)\end{array}$ & $\begin{array}{c}0 \\
(0)\end{array}$ \\
\hline$C(n=24)$ & $\begin{array}{c}21 \\
(10.8 \%)\end{array}$ & $\begin{array}{c}20 \\
(10.3 \%)\end{array}$ & $\begin{array}{c}22 \\
(11.3 \%)\end{array}$ & $\begin{array}{c}17 \\
(8.8 \%)\end{array}$ & $\begin{array}{c}18 \\
(9.3 \%)\end{array}$ & $\begin{array}{c}12 \\
(6.2 \%)\end{array}$ & $\begin{array}{c}3 \\
(1.5 \%)\end{array}$ & $\begin{array}{c}24 \\
(12.4 \%)\end{array}$ & $\begin{array}{c}1 \\
(0.5 \%)\end{array}$ & $\begin{array}{c}0 \\
(0)\end{array}$ \\
\hline AK $(n=5)$ & $\begin{array}{c}4 \\
(2 \%)\end{array}$ & $\begin{array}{c}2 \\
(1 \%)\end{array}$ & $\begin{array}{c}4 \\
(2 \%)\end{array}$ & $\begin{array}{c}5 \\
(2.6 \%)\end{array}$ & $\begin{array}{c}3 \\
(1.5 \%)\end{array}$ & $\begin{array}{c}3 \\
(1.5 \%)\end{array}$ & $\begin{array}{c}2 \\
(1 \%)\end{array}$ & $\begin{array}{c}1 \\
(0.5 \%)\end{array}$ & $\begin{array}{c}5 \\
(2.6 \%)\end{array}$ & $\begin{array}{c}0 \\
(0)\end{array}$ \\
\hline $\mathrm{I}(\mathrm{n}=0)$ & $\begin{array}{c}0 \\
(0)\end{array}$ & $\begin{array}{c}0 \\
(0)\end{array}$ & $\begin{array}{c}0 \\
(0)\end{array}$ & $\begin{array}{c}0 \\
(0)\end{array}$ & $\begin{array}{c}0 \\
(0)\end{array}$ & $\begin{array}{c}0 \\
(0)\end{array}$ & $\begin{array}{c}0 \\
(0)\end{array}$ & $\begin{array}{c}0 \\
(0)\end{array}$ & $\begin{array}{c}0 \\
(0)\end{array}$ & $\begin{array}{c}0 \\
(0)\end{array}$ \\
\hline
\end{tabular}


Table 3. Antimicrobial patterns among multiple-Antibiotic resistant (MAR) isolates of E.coli

\begin{tabular}{|c|c|c|c|}
\hline \multirow[t]{2}{*}{ Sampling Point } & \multirow[t]{2}{*}{ Date } & \multicolumn{2}{|c|}{ Escherichia coli } \\
\hline & & o. of isolates & MAR pattern \\
\hline \multirow[t]{11}{*}{ 1. Station } & January 2009 & 1 & {$[\mathrm{AM}],[\mathrm{CAZ}],[\mathrm{NA}]$} \\
\hline & February 2009 & 1 & {$[\mathrm{AMC}, \mathrm{AM}]^{*},[\mathrm{NA}],[\mathrm{SXT}]$} \\
\hline & September 2009 & 1 & {$[\mathrm{AMC}, \mathrm{AM}]^{*},[\mathrm{TE}],[\mathrm{NA}]$} \\
\hline & April 2009 & 1 & {$[\mathrm{AMC}, \mathrm{AM}]^{*},[\mathrm{NA}],[\mathrm{SXT}],[\mathrm{S}]$} \\
\hline & October 2009 & 1 & {$[\mathrm{AMC}, \mathrm{AM}] *,[\mathrm{TE}],[\mathrm{S}],[\mathrm{C}]$} \\
\hline & April 2010 & 1 & {$[\mathrm{AM}],[\mathrm{CAZ}],[\mathrm{TE}],[\mathrm{NA}],[\mathrm{AK}]$} \\
\hline & January, May 2010 & 2 & {$[\mathrm{C}],[\mathrm{SXT}],[\mathrm{S}],[\mathrm{NA}],[\mathrm{AMC}, \mathrm{AM}] *,[\mathrm{TE}],[\mathrm{CAZ}]$} \\
\hline & March 2011 & 1 & {$[\mathrm{AMC}, \mathrm{AM}]^{*},[\mathrm{SXT}]$} \\
\hline & October 2011 & 1 & {$[\mathrm{C}],[\mathrm{S}],[\mathrm{AMC}, \mathrm{AM}]^{*},[\mathrm{TE}],[\mathrm{NA}]$} \\
\hline & November 2011 & 1 & {$[\mathrm{C}],[\mathrm{SXT}],[\mathrm{AMC}, \mathrm{AM}]^{*},[\mathrm{TE}]$} \\
\hline & December 2011 & 1 & {$[\mathrm{~S}],[\mathrm{SXT}],[\mathrm{NA}],[\mathrm{AMC}, \mathrm{AM}]^{*},[\mathrm{TE}]$} \\
\hline \multirow[t]{11}{*}{ 2. Station } & October 2009 & 1 & {$[\mathrm{SXT}],[\mathrm{NA}],[\mathrm{AMC}, \mathrm{AM}]^{*},[\mathrm{TE}]$} \\
\hline & July 2009 & 1 & {$[\mathrm{TE}],[\mathrm{AMC}],[\mathrm{CAZ}]$} \\
\hline & August, November 2009 & 2 & {$[\mathrm{C}],[\mathrm{S}],[\mathrm{SXT}],[\mathrm{NA}],[\mathrm{AMC}, \mathrm{AM}]^{*},[\mathrm{TE}]$} \\
\hline & March 2009 & 1 & {$[\mathrm{C}],[\mathrm{AK}, \mathrm{S}]^{*},[\mathrm{SXT}],[\mathrm{NA}],[\mathrm{AMC}, \mathrm{AM}]^{*},[\mathrm{TE}]$} \\
\hline & May, August 2010 & 2 & {$[\mathrm{SXT}],[\mathrm{NA}],[\mathrm{AMC}, \mathrm{AM}]^{*},[\mathrm{TE}]$} \\
\hline & September 2010 & 1 & {$[\mathrm{SXT}],[\mathrm{NA}],[\mathrm{AMC}, \mathrm{AM}]^{*},[\mathrm{TE}],[\mathrm{C}]$,} \\
\hline & February 2010 & 1 & {$[\mathrm{SXT}],[\mathrm{NA}],[\mathrm{AMC}, \mathrm{AM}] *,[\mathrm{TE}],[\mathrm{C}],[\mathrm{S}]$} \\
\hline & December 2010 & 1 & {$[\mathrm{SXT}],[\mathrm{NA}],[\mathrm{AMC}, \mathrm{AM}]^{*},[\mathrm{TE}],[\mathrm{C}],[\mathrm{S}],[\mathrm{CAZ}]$} \\
\hline & January 2011 & 1 & {$[\mathrm{AMC}, \mathrm{AM}]^{*},[\mathrm{SXT}],[\mathrm{CAZ}]$} \\
\hline & April 2011 & 1 & {$[\mathrm{C}],[\mathrm{S}],[\mathrm{AMC}, \mathrm{AM}]^{*},[\mathrm{SXT}],[\mathrm{NA}],[\mathrm{TE}]$} \\
\hline & October 2011 & 1 & {$[\mathrm{~S}],[\mathrm{AMC}, \mathrm{AM}]^{*},[\mathrm{SXT}],[\mathrm{NA}],[\mathrm{TE}]$} \\
\hline \multirow[t]{14}{*}{ 3. Station } & February 2009 & 1 & {$[\mathrm{AMC}, \mathrm{AM}]^{*},[\mathrm{TE}]$} \\
\hline & March 2009 & 1 & {$[\mathrm{SXT}],[\mathrm{NA}],[\mathrm{AM}]$} \\
\hline & July 2009 & 1 & {$[\mathrm{AMC}, \mathrm{AM}]^{*},[\mathrm{CAZ}]$} \\
\hline & May 2009 & 1 & {$[\mathrm{~S}],[\mathrm{SXT}],[\mathrm{AM}],[\mathrm{TE}]$} \\
\hline & August 2009 & 1 & {$[\mathrm{AMC}, \mathrm{AM}]^{*},[\mathrm{SXT}],[\mathrm{NA}],[\mathrm{TE}]$} \\
\hline & October 2010 & 1 & {$[\mathrm{AMC}, \mathrm{AM}]^{*},[\mathrm{NA}]$} \\
\hline & February, November 2010 & 2 & {$[\mathrm{SXT}],[\mathrm{AMC}, \mathrm{AM}] *[\mathrm{TE}]$} \\
\hline & February 2010 & 1 & {$[\mathrm{SXT}],[\mathrm{AMC}, \mathrm{AM}]^{*},[\mathrm{NA}]$} \\
\hline & August 2010 & 1 & {$[\mathrm{AK}, \mathrm{S}] *,[\mathrm{SXT}],[\mathrm{NA}],[\mathrm{AMC}, \mathrm{AM}] *$} \\
\hline & September 2010 & 1 & {$[\mathrm{SXT}],[\mathrm{NA}],[\mathrm{AMC}, \mathrm{AM}]^{*},[\mathrm{C}],[\mathrm{TE}]$} \\
\hline & January 2011 & 1 & {$[\mathrm{AMC}, \mathrm{AM}]^{*},[\mathrm{C}],[\mathrm{TE}]$} \\
\hline & February 2011 & 1 & {$[\mathrm{AMC}, \mathrm{AM}]^{*},[\mathrm{TE}]$} \\
\hline & May 2011 & 1 & {$[\mathrm{AMC}, \mathrm{AM}]^{*},[\mathrm{TE}],[\mathrm{SXT}]$} \\
\hline & December 2011 & 1 & {$[\mathrm{AMC}, \mathrm{AM}]^{*},[\mathrm{SXT}],[\mathrm{NA}],[\mathrm{TE}]$} \\
\hline \multirow[t]{13}{*}{ 4. Station } & January 2009 & 1 & [S], [SXT], [TE], [AM] \\
\hline & March 2009 & 1 & {$[\mathrm{~S}],[\mathrm{SXT}],[\mathrm{AM}],[\mathrm{NA}]$} \\
\hline & April 2009 & 1 & {$[\mathrm{AMC}, \mathrm{AM}]^{*},[\mathrm{NA}]$} \\
\hline & June 2009 & 1 & {$[\mathrm{AMC}, \mathrm{AM}]^{*},[\mathrm{NA}],[\mathrm{CAZ}]$} \\
\hline & October 2009 & 1 & {$[\mathrm{AMC}, \mathrm{AM}]^{*},[\mathrm{~S}],[\mathrm{SXT}],[\mathrm{NA}],[\mathrm{TE}]$} \\
\hline & April 2010 & 1 & {$[\mathrm{AMC}, \mathrm{AM}]^{*},[\mathrm{TE}]$} \\
\hline & July 2010 & 1 & {$[\mathrm{AMC}, \mathrm{AM}]^{*},[\mathrm{SXT}]$} \\
\hline & May 2010 & 1 & {$[\mathrm{AMC}, \mathrm{AM}]^{*},[\mathrm{~S}],[\mathrm{SXT}],[\mathrm{TE}]$} \\
\hline & January, July 2011 & 2 & {$[\mathrm{AMC}, \mathrm{AM}]^{*},[\mathrm{SXT}],[\mathrm{TE}],[\mathrm{NA}]$} \\
\hline & March 2011 & 1 & {$[\mathrm{C}],[\mathrm{SXT}],[\mathrm{AMC}, \mathrm{AM}]^{*},[\mathrm{TE}]$} \\
\hline & September 2011 & 1 & {$[\mathrm{AMC}, \mathrm{AM}]^{*},[\mathrm{TE}],[\mathrm{NA}]$} \\
\hline & November 2011 & 1 & {$[\mathrm{AMC}, \mathrm{AM}]^{*},[\mathrm{TE}],[\mathrm{NA}],[\mathrm{C}],[\mathrm{SXT}]$} \\
\hline & December 2011 & 1 & {$[\mathrm{~S}],[\mathrm{SXT}],[\mathrm{TE}]$} \\
\hline \multirow[t]{2}{*}{ 5. Station } & April 2009 & 1 & {$[\mathrm{C}],[\mathrm{SXT}],[\mathrm{AM}],[\mathrm{TE}],[\mathrm{NA}]$} \\
\hline & June 2009 & 1 & [S], [SXT], [AM], [TE], [CAZ] \\
\hline
\end{tabular}




$\begin{array}{ll}\text { October 2009 } & 1 \\ \text { November 2009 } & 1 \\ \text { March 2009 } & 1 \\ \text { December 2009 } & 1 \\ \text { January, September 2010 } & 2 \\ \text { May 2010 } & 1 \\ \text { November, December 2010 } & 2 \\ \text { March 2010 } & 1 \\ \text { April 2010 } & 1 \\ \text { February 2010 } & 1 \\ \text { January 2011 } & 1 \\ \text { May 2011 } & 1 \\ \text { September 2011 } & 1 \\ \text { November 2011 } & 1 \\ \text { December 2011 } & 1 \\ \text { February 2010 } & 1 \\ \text { May 2010 } & 1 \\ \text { June 2010 } & 1 \\ \text { November 2010 } & 1 \\ \text { December 2010 } & 1 \\ \text { January 2011 } & 1 \\ \text { October 2011 } & 1 \\ \text { November 2011 } & 1 \\ \text { January, December 2010 } & 2 \\ \text { June 2010 } & 1 \\ \text { September 2010 } & 1 \\ \text { May 2010 } & 1 \\ \text { October 2010 } & 1 \\ \text { November 2010 } & 1 \\ \text { January 2011 } & 1 \\ \text { December 2011 } & 1 \\ \end{array}$

[AMC, AM]*, [TE], [NA], [CAZ]

[AMC, AM]*, [TE], [NA], [S], [SXT]

[AMC, AM]*, [TE], [NA], [S], [SXT],[C]

$[\mathrm{AMC}, \mathrm{AM}]^{*},[\mathrm{TE}],[\mathrm{NA}],[\mathrm{S}],[\mathrm{SXT}],[\mathrm{CAZ}]$

[AMC, AM]*, [NA]

$[\mathrm{AMC}, \mathrm{AM}]^{*},[\mathrm{TE}],[\mathrm{CAZ}]$

[AMC, AM]*, [TE], [NA]

[AMC, AM]*, [TE], [NA], [SXT]

[AMC, AM]*, [TE], [S], [SXT]

[AMC, AM]*, [TE], [NA], [S], [SXT]

[NA], [SXT], [AMC], [TE]

[AMC, AM]*, [NA], [SXT], [CAZ]

[AMC, AM]*, [TE], [NA]

[AMC, AM]*, [NA], [TE], [SXT], [C]

[AMC, AM]*, [SXT], [S], [TE]

[AK, S]*, [SXT], [NA], [TE], [AM], [CAZ]

[AMC, AM]*, [TE]

[S], [SXT], [NA], [TE], [AM], [CAZ]

[NA], [TE], [AM]

[SXT], [AMC, AM]*, [TE]

[AMC, AM]*, [NA]

[AMC, AM]*, [NA], [TE], [SXT], [C]

[SXT], [C], [TE]

$[\mathrm{AMC}, \mathrm{AM}]^{*},[\mathrm{TE}]$

[AMC, AM]*, [SXT]

[AMC, AM]*, [NA]

[AMC, AM]*, [NA] ,[SXT], [CAZ]

$[\mathrm{AMC}, \mathrm{AM}]^{*},[\mathrm{NA}],[\mathrm{SXT}]$

[C], [AMC, AM]*, [TE], [NA]

[AMC, AM]*, [NA], [TE], [SXT]

[AMC, AM]*, [NA], [TE], [SXT], [S], [CAZ]

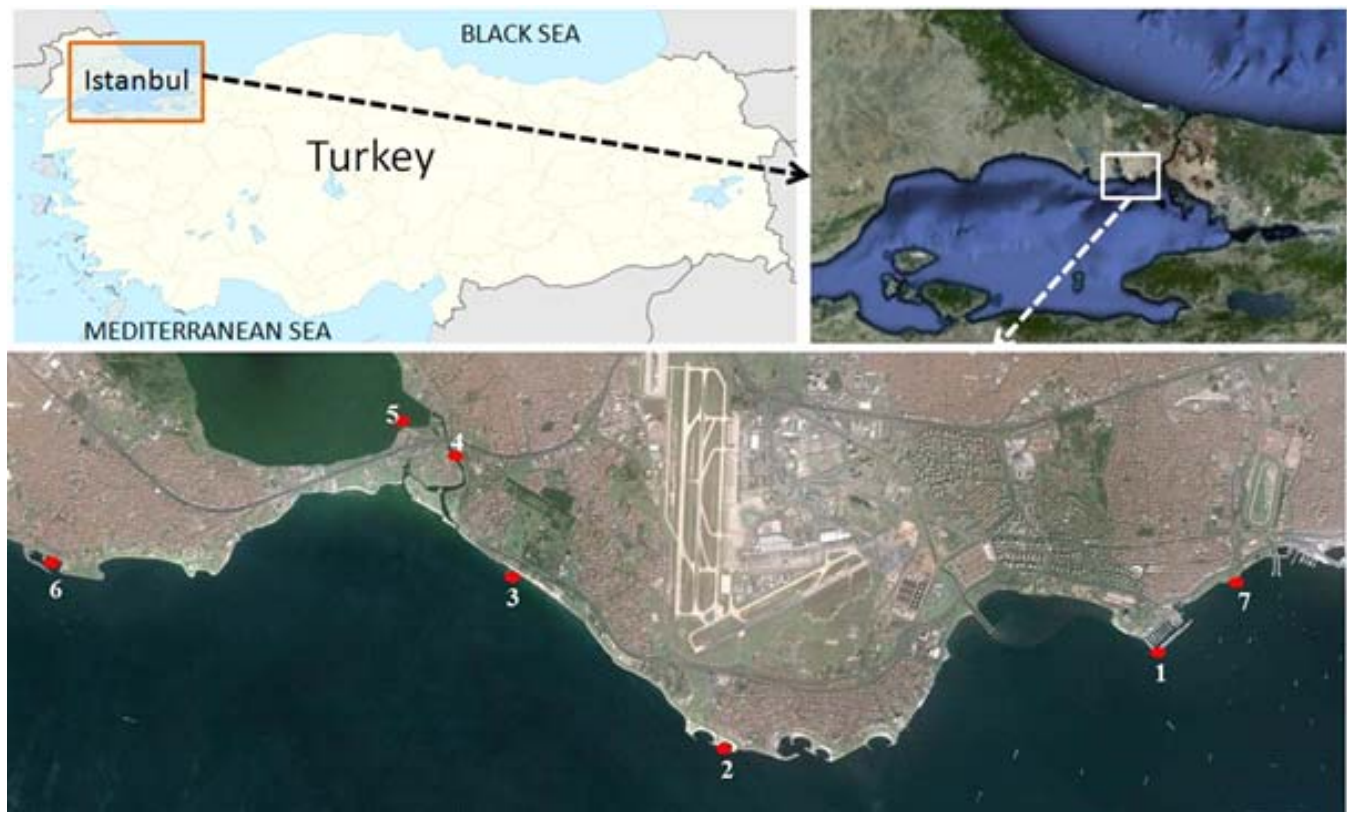

Fig. 1. Study Area 


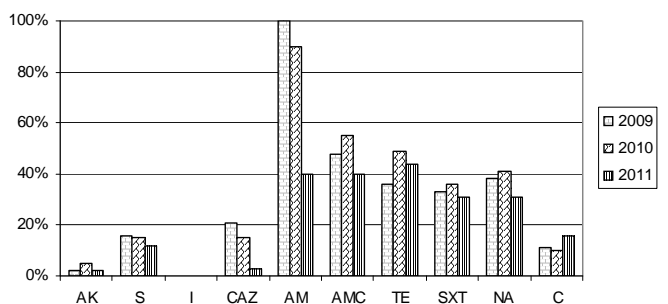

Fig. 2. Antimicrobial susceptibility of E.coli strains

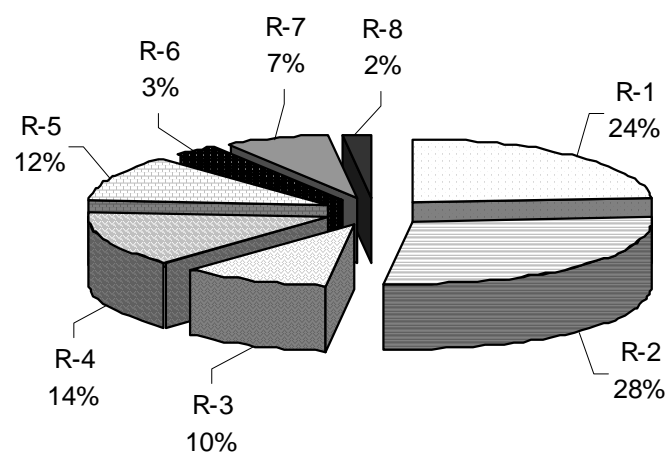

Fig. 3. Distribution of antimicrobial patterns among E.coli isolates resistant to analyzed antimicrobial agents for 2009 (R1: resistant to one antibiotic, R8 : resistant to eight antibiotic)

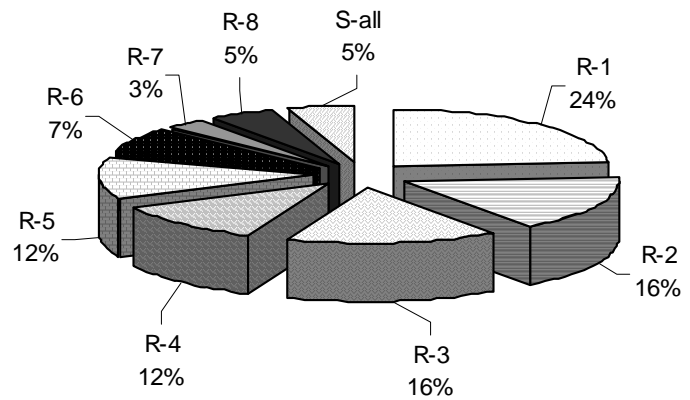

Fig. 4. Distribution of antimicrobial patterns among E.coli isolates sensitive to all (S-all) and resistant to analyzed antimicrobial agents for 2010 (R1: resistant to one antibiotic, R8 : resistant to eight antibiotic)

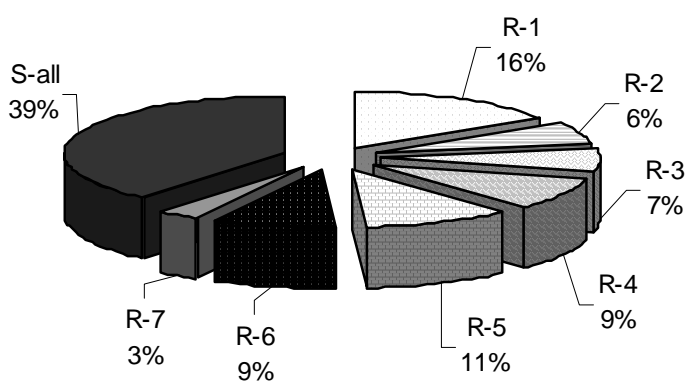

Fig. 5. Distribution of antimicrobial patterns among E.coli isolates sensitive to all (S-all) and resistant to analyzed antimicrobial agents for 2011 (R1: resistant to one antibiotic, R8 : resistant to eight antibiotic) at least one antibiotic and 24\% showed resistance to 5 or more antibiotics (Figure 3).

In 2010, of the E.coli analyzed, all strains except $5 \%$ showed resistance to at least one antibiotic and $28 \%$ showed resistance to 5 or more (R5+R6+R7+R8) antibiotics (Figure 4). In 2011, of the E.coli analyzed, all strains except 39\% showed resistance to at least one antibiotic and 23\% showed resistance to 5 or more antibiotics (Figure 5).

While ampicillin resistance was the highest seen resistance throughout the study, the most commonly seen resistance pairing in all the years were AM-AMC, AM-TE, AM-NA, and AMSXT with ratios of $45.9 \%$, 38.1\%, 32\%, and 31\% respectively. When compared with other antibiotic pairings, the highest resistance was seen in AMCTE antibiotic at 31\%, which was followed by the ratios for AMC-NA(27.3\%) and AMC-SXT (26.3\%).

In the study, the lowest resistance rates were seen with AK-C antibiotic pairings (0.5\%), which was followed by AK-AMC and AK-CAZ at $1 \%$ and AK-SXT, AK-S, and C-CAZ at $1.5 \%$. Table 3 shows the resistance phenotypes for E.coli strains for more than one type of antibiotic.

\section{DISCUSSION}

Antibiotic resistance is an extremely serious problem that is widespread throughout the world and commonly seen during disease treatment ${ }^{4}$. With the increase in antibiotic resistance in the E.coli bacteria, national and international organizations not only need to increase the amount of studies conducted but also deem the re-evaluation of multiple antibiotic resistance as an obligatory practice ${ }^{5,6,11}$. Studying antibiotic resistance in bacteria found in shoreline waters is important in terms of explaining the relationship between human activities and shoreline ecosystems ${ }^{6,17}$.

In this study, 194 strains of E.coli were isolated, in which ampicillin (74.4\%) and amoxicillin (47.4\%) had the highest resistance rates. Tetracycline resistance was found to be $43.3 \%$. While $84.4 \%$ of the isolates were found to be resistant to at least one or more antibiotic, 63.4\% were resistance to 2 or more antibiotics and $24.7 \%$ were resistant to 5 or more antibiotics.

In their study conducted in the eastern 
harbor in Egypt, they found multiple antibiotic resistances in the bacteria they isolated from sea water and also found that this resistance increased in parallel with heavy antibiotic use $\mathrm{e}^{18}$. In another study conducted in Germany the antibiotic resistance characteristics of E.coli strains isolated from cattle, pig and barnyard fowl were analyzed. All strains showed resistance to one (8\%) or more than one (32\%) antibiotic with the lowest resistance rates being seen in the cattle isolates. In addition, resistance to sulfamethoxazole, tetracycline, and streptomycin was between $28 \%$ - 30\%, ampicillin and spectinomicin was between 15\%-19\%, and nalidixic acid, canamycine, trimethoprim, neomicin, trimethoprim-sulfamethoxazole, and chloramphenicol was between $8 \%-\% 11$. As a result, the study concluded that antimicrobial agents should be used much more cautiously in animal farms ${ }^{7}$.

Studies conducted in Turkey have found heavy antibiotic use resulting in multiple resistance (MAR) index values being $>0.2$ and heavy levels of human and animal based contamination sources in various areas ${ }^{10}$. In a study conducted in the Izmit Bay, Cingilli-Vural and Akçin found antibiotic resistances for E.coli strains to be $50 \%$ for tetracycline, $62.5 \%$ for sulbactam/ampicillin, $62.5 . \%$ for penicillin, $12.5 \%$ for amicasin, $37.5 \%$ for chloramphenicol, and $62.5 \%$ for trimethoprimsulfamethoxazole ${ }^{19}$. Ozgumus et al., examined seawater based coliforms class 1 and class 2 integron gene tapes and antibiotic resistance characteristics. The study found that ampicillin resistant E.coli contained the TEM- 1 type $\beta$ lactamase gene ${ }^{20}$. Also, they found that among the tetracycline resistant strains, the tet (B) gene was common. In addition to this, it was also found that the resistance against ampicillin, tetracycline, and streptomycin were transferable. The finding suggests that because antibiotic resistant coliforms exist in sea water, they may pose risks for both public and environmental health. In this study, despite the fact that tetracycline was not an antibiotic used in the treatment of E.coli infections, it showed resistance which can be possibly linked to the idea that resistance determinants, among the same or different types of enteric bacteria, tend to spread rapidly.

In this study, all isolates were found to be sensitive to the antibiotic imipenem; however,
Toroðlu et al., found that 27 (40\%) of the gram negative bacteria they isolated from the Aksu River were resistant to 5 or more antibiotics and that in $49.3 \%$ of the isolates, â-lactamase production was determined ${ }^{21}$. In the study, while all of the strains showed no resistance towards cefotaxime, the formation of resistance against antibiotics meropenem and imipenem were striking.

\section{CONCLUSIONS}

The finding that the E.coli strains, isolated from Istanbul's south-west shoreline, carried high levels of multiple antibiotic resistances was consistent with previous studies conducted in water based locations. In this study, the largest antibiotic resistance seen was in the penicillin group, which is widely used in the treatment of many infections and associated with the following factors: they have been used since the 1940's, they have bactericide properties, they have low levels of toxicity, they are cheap, and they are sensitive in producing effective results to bacterial infections ${ }^{22}$. The second highest resistant class was seen in the tetracycline group, which has been used for many years in the treatment for human infections, breeding and veterinary medicine. Today, resistance against tetracycline seen in many bacteria is thought to be the end result of widespread antibiotic use ${ }^{23}$.

In this study, no resistance was detected in the antibiotic imipenem, which is a carbapenem derivate that was the first type of antibiotic to be used clinically. For this reason, it can be said that the antibiotic imipenem is highly resistant due to a resistance mechanism supported by â-lactamase, which is commonly seen in E.coli strains (24). With the number of the resistant strains out numbering the number of sensitive strains, serious concerns regarding antibiotic resistance in sea water based bacteria are raised.

As a result, the association between wrong and insufficient refinement and pollution indicator bacteria spreading throughout the environment is clear. It is worrying that the vast majority of E. coli strains isolated from at least resistance one antibiotic as well as to show multiple antibiotic resistance seen in many strains. In addition antibiotic resistant strains of bacteria of marine origin are much more than susceptible 
strains. Multiple antibiotic resistant (MAR) indices calculated to assess health risks due to the presence of resistant E.coli suggested an increased presence of antibiotics in surface water, likely from anthropogenic sources as no other wastewater contributions in the area were documented. These findings, which were obtained throughout Istanbul's shores which are frequently used for recreation, fishing, and transport, show that public health is under serious risks.

\section{ACKNOWLEDGEMENTS}

This study was conducted with the support of the Research Fund of Istanbul University, Research Project Number 18373. The authors special thank Ms. Nevran Sultan Sisli, Ms. Devran Algur and CEMIK Team for their assistance with field work. Assist. Prof. Dr. N. Linda Fraim commented on an earlier draft of this manuscript.

\section{REFERENCES}

1. MARTINEZ JL. Environmental pollution by antibiotics and by antibiotic resistance determinants. Environmental Pollution. 157: 2893-2902, 2009.

2. Watkinson Aj, Micalizzi Gb, Graham Gm, Bates $\mathrm{Jb}$, Costanzo Sd. Antibiotic-Resistant Escherichia coli in Wastewaters, Surface Waters, and Oysters from an Urban Riverine System. Applied and Enviromental Microbiology, 73 : 5667-5670, 2007.

3. Mackie RI, Koike S, Krapac I, Chee-Sanford J, Maxwell S, Aminov RI. Tetracycline Residues and Tetracycline Resistance Genes in Groundwater Impacted by Swine Production Facilities. Animal Biotechnology. 17: 157-176, 2006.

4. Danishta I, Ismet M, Sonatun D, JaufeerallyFakim Y. Antibiotic Resistance of Escherichia coli Isolates from Environmental and Waste Water Samples in Mauritius. Advances in Environmental Biology. 4: 1-9, 2010.

5. Sivri N, Seker DZ. Investigation of Enteric Bacteria of Surface Waters in the Southwestern Coast of Istanbul by means of GIS, Turkish Journal of Fisheries and Aquatic Sciences. 10: 505-511, 2010.

6. Sivri N, Sandalli C, Ozgumus OB, Colakoglu F, Dogan D. Antibiotic Resistance Profiles of Enteric Bacteria Isolated from Kucukcekmece Lagoon (Istanbul-Turkey). Turkish Journal of
Fisheries and Aquatic Sciences. 12: 699-707, 2012.

7. Guerra B, Junker E, Schroeter A, Malorny B, Lehmann S, Helmuth R. Phenotypic and genotypic characterization of antimicrobial resistance in German Escherichia coli isolates from cattle, swine and poultry. Journal of Antimicrobial Chemotherapy. 52: 489-492, 2003.

8. Alhaj N, Mariana NS, Raha AR, Ishak, Z. Prevalence of Antibiotic Resistance among Escherichia coli from Different Sources in Malaysia. International Journal of Poultry Science. 6:293-297, 2007.

9. Morse A \& Jackson A. Fate of a Representative Pharmaceutical in the Environment, Thesis (PhD), Texas Tech University Texas Water Resources Institute. 2003.

10. Matyar F, Kaya A, Dinçer S. Antibacterial Agents and Heavy Metal Resistance in GramNegative Bacteria Isolated from Seawater, Shrimp and Sediment in Iskendurun Bay, Turkey. Science of the Total Enviroment. 407: 279-285, 2008.

11 Von Baum H \& Marre R. Antimicrobial Resistance of Escherichia coli and Therapeutic Ýmplications. International Journal of Medical Microbiology. 295: 503-511, 2005.

12. Morris DW. The Application of Multiple Antibiotic Resistance Profiles of Coliforms to Detect Sources of Bacterial Contamination of the Anacostia River. Final Report. District of Columbia Water Resources Research Institute. 29. 2011.

13. American Public Health Association (APHA), American Water Works Association (AWWA) and Water Pollution Control Federation. Standard methods for the examination of water and wastewater. 21st Ed., Washington. 2005.

14. Sivri, N., Allen, M.J., Jones, M., Akbulut, V. Potential Public Health Significance of Faecal Contamination in South-western Coastal Area in Istanbul, Turkey, Journal of Pure And Applied Microbiology (JPAM), 8(5), 3789-3796, 2014.

15. Erturk, A., Sivri, N., Seker, D.Z., Gurel, M., Ozman Say, A.N., Tanik, A. Ozturk, I. Analysis of the Distribution of Phytoplankton and Enteric Bacteria in Efteni Lake, Turkey, African Journal of Microbiology Research, 8 (21) 21442154, 2014.

16. CLSI - Clinical and Laboratory Standards Institute (CLSI). Performance standards for antimicrobial disk susceptibility tests; 8th ed., Approved standard M2-A8., CLSI, Wayne, PA. 2003.

17. AUERBACH EA, SEYFRIED EE, McMahon 
KD. Tetracycline Resistance Genes in Activated Sludge Wastewater Treatment Plants. Water Research. 41: 1143-1151, 2007.

18. Sabry SA, Ghozlan HA, Abou-ZeId DM. Metal Tolerance and Antibiotic Resistance Patterns of a Bacterial Isolated from Sea water. J. App. Mic. 82: 245-252, 1997.

19. Cingilli-Vural H, Akçin A. Investigation of "Contagious Type Antibiotic Resistance Properties” Related with R Plasmids in Escherichia coli Strains Isolated from Ýzmit Gulfs (Turkey). Kafkas Univ Vet Fak Derg. 17 : 23-30, 2011.

20. Ozgumus OB, Sandalli C, Sevim A, Celik-Sevim E., Sivri N. Class 1 and class 2 integrons and plasmid-mediated antibiotic resistance in coliforms isolated from ten rivers in northern
Turkey. J. Microbiol. 47: 19-27, 2009.

21. Toroðlu S, Dinçer S, Korkmaz H. Antibiotic Resistance in Gram Negative Bacteria Isolated from Aksu River in (Kahramanmarap) Turkey. Annuals of Microbiology. 55: 229-233, 2005.

22. Kummerer K. Antibiotics in the Aquatic Environment - A Review - Part I. Chemosphere. 75: 417-434, 2009.

23. Chopra I, Roberts M. Tetracycline Antibiotics: Mode of Action, Applications, Molecular Biology, and Epidemiology of Bacterial Resistance. Microbiology and Molecular Biology Reviews. 65 : 232-60, 2001.

24. LIvermore DM, Woodford N. Carbapenemases: a Problem in Waiting? Current Opinion Microbiology. 3: 489-495, 2000. 